\title{
ESTUDO DA UTILIZAÇÃO DA ESTRUTURA DE CONTENÇÃO TIPO GABIÃO
}

\section{STUDY OF THE USE OF STRUCTURE OF CONTAINMENT GABION TYPE}

Lucilene Boniolo da Cruz, Maíra Ferreira Lima Braghin

Universidade do Oeste Paulista - UNOESTE, Faculdade de Engenharias e Arquitetura e Urbanismo, Presidente Prudente, SP.

E-mail: lucilene_boniolo@hotmail.com

RESUMO - O presente trabalho, intitulado "Estudo da utilização da estrutura de contenção tipo gabião", teve como objetivo fazer uma análise sobre a viabilidade desse tipo de muro, considerando suas características físicas, comparando-o economicamente com outros muros, e com visitas às obras de gabiões em Presidente Prudente - SP. Sua metodologia, foi baseada em livros, tabelas orçamentárias, programas de dimensionamento e comparativos de custos entre três tipos de muros. Os resultados, mostraram que em Presidente Prudente, os gabiões foram utilizados como contenções de taludes próximos a pontes e aterros de terra. No dimensionamento, mostrou resistência ao empuxo ativo. Já no comparativo de custo, ele foi o segundo mais econômico. Por fim, chegou-se à conclusão que o muro gabião é mais vantajoso quando aplicado em taludes com crescimento de vegetação, que ele possuiu alta resistência e, embora não tenha sido o mais economicamente barato, alguns fatores influenciam em sua escolha para o uso.

Palavras-chave: Gabião; Estrutura de Contenção; Muros; Presidente Prudente; Aterros De Terra.

ABSTRACT - This study, entitled "Study of the use of the containment structure type gabion", aimed to make an analysis of the viability of this type of wall, considering its physical characteristics, comparing the cost with other walls, and visits gabions workings in Presidente Prudente - SP. The methodology is based on books, tables budget, dimensioning programs and comparative costs between three types of walls. The results showed that in Presidente Prudente, the gabions were used as containment embankments near bridges and earth embankments. In dimensioning, showed resistance to the active thrust. In the cost comparison, it was the second most economical. Finally, come to the conclusion that the wall gabion is more advantageous when applied to slopes with vegetation growth, which

Recebido em: $17 / 08 / 2016$ Revisado em: 22/08/2016 Aprovado em: 23/08/2016 he possessed high strength and, although it was not the most economically cheap, some factors influence its choice for use.

Keywords: Gabion; Containment Structure; Walls; Presidente Prudente; Earth Embankments. 


\section{INTRODUÇÃO}

A estrutura de contenção do solo, conhecida popularmente também como muro de arrimo, faz parte das mais antigas construções da humanidade. Ela tem como função, a contenção de um maciço de terra ou de rochas, transmitindo seus esforços para solo e proporcionando estabilidade, garantindo a segurança do que existe acima e abaixo delas (GERSCOVICH, 2007).

Segundo Moliterno, (1980), existe atualmente vários tipos de estruturas de contenção, com diferentes formas, tamanhos e oferecendo o emprego de diversos tipos de matérias. De acordo com Barros (2008) o tipo gabião, é um muro de gravidade flexível constituídos de elementos metálicos feitos com telas de malha hexagonal de dupla torção, preenchidos com pedras que podem ser seixos rolados, pedras basálticas e granito.

Foi observado que na cidade de Presidente Prudente -SP, são poucas as obras que utilizam o tipo gabião, e com a intenção de estimular a utilização do muro gabião na cidade, objetiva-se com esse trabalho, fazer uma análise de como a estrutura é utilizada em Presidente Prudente-SP, um teste de dimensionamento utilizando o programa GEO5, desenvolvido pela empresa FINE CIVIL ENGINEERING SOFTWARE, e um comparativo de custo do muro gabião com outros dois tipos de muros de contenção.

\section{PRIMEIRA PÁGINA}

Em sua metodologia, foram feitas visitas in loco de muros de gabião na cidade de Presidente Prudente - São Paulo, analisando-os visivelmente sua relação com a paisagem local, como foram construídos e para quais finalidades. Em seguida, foi realizado um teste de dimensionamento utilizando o programa de software GEO5, fazendo o uso do solo do tipo argila arenosa, de consistência firme, com as características descritas na Figura 1. Foi considerado um talude com altura de $4 \mathrm{~m}$ com ângulo de inclinação de $\beta 22$, junto com uma carga permanente de $5 \mathrm{KN} / \mathrm{m}^{2}$ sobre o terreno.

$\begin{array}{ll}\text { Argila arenosa (CS), consistência firme } \\ \text { Peso espećfio: } & \gamma=18,50 \mathrm{kN} / \mathrm{m}^{3} \\ \text { Estado de tensão : } & \text { efetivo } \\ \text { Ângulo de atrito interno: } & \text { Qef }=24,50^{\circ} \\ \text { Coesão do solo : } & c_{\text {ef }}=14,00 \mathrm{kPa} \\ \text { Ângulo de atrito estru. -solo: } & \delta=0,00^{\circ} \\ \text { Solo: } & \text { coesivo } \\ \text { Coeficiente de Poisson : } & \mathrm{v}=0,35 \\ \text { Peso espećfico saturado : } & \eta_{\text {sat }}=18,50 \mathrm{kN} / \mathrm{m}^{3}\end{array}$

Figura 01. Características do solo utilizado para teste. Fonte: GEO5 (2016) 
Para as dimensões do muro, foi adotado um escalonamento do lado externo. Para cada 0,5m na horizontal, subiu-se $1 \mathrm{~m}$ na

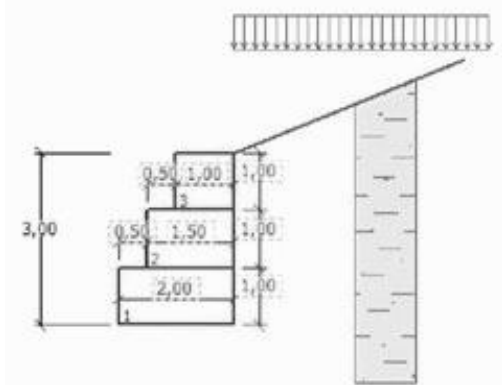

vertical, até que se totalizou uma altura de $3 m$ com uma base de $2 \mathrm{~m}$. O desenho esquemático do muro está na Figura 2.

Figura 02. Dimensões adotadas para verificação da estrutura.

Fonte: GEO5 (2016)

Por último, foi feito um comparativo de custo baseado na tabela da SINAP (Sistema Nacional de Pesquisa de Custos e Índices da Construção Civil), considerando os muros gabião, o concreto ciclópico e o de alvenaria de pedra.

\section{RESULTADOS}

Todos os muros visitados foram construídos para contenções de solos e de taludes as margens de córregos. Como por exemplo, um localizado no campus II da
Universidade do Oeste Paulista (UNOESTE) em Presidente Prudente - São Paulo, construído em 2013 próximo ao córrego do Limoeiro, com a função de sustentar o talude.

Segundo o projeto, foram utilizados gabiões em formatos de caixas escalonadas para o lado de dentro do solo. O muro possui uma altura de 5 metros e uma base de 3,5 metros, como é possível ver no corte em projeto, realizado pela empresa MACCAFERRI, responsável pela obra. 


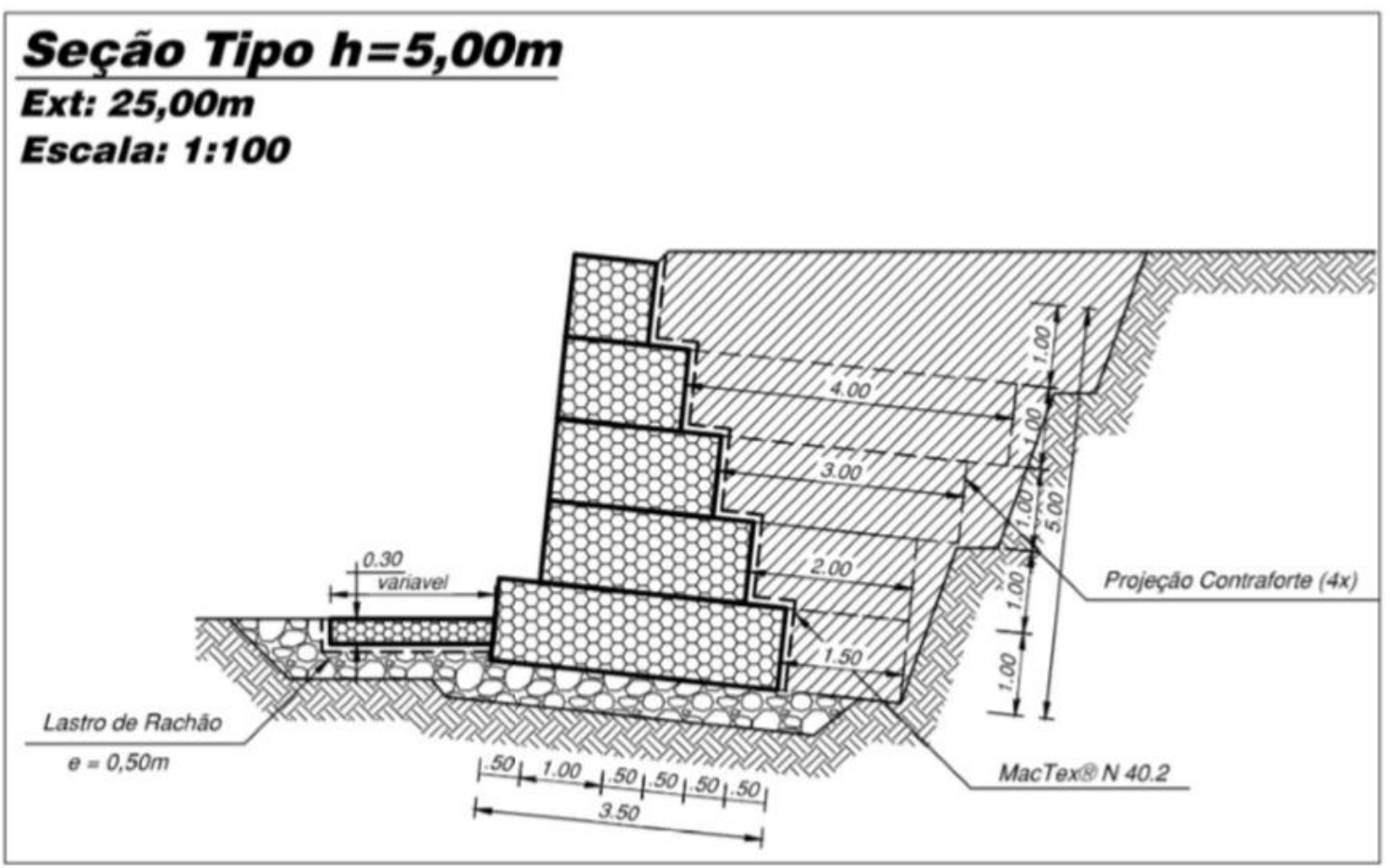

Figura 03. Seção em corte do muro construído na Universidade do Oeste Paulista. Fonte: (Arquivo MACCAFERRI AMÉRICA LATINA, adaptado pelos autores, 2016)

Devido aos espaços vazios gerados fornecendo até mesmo um auxílio na pelos tamanhos desiguais das pedras, a drenagem do solo pelos espaços existentes. A vegetação encontrou espaço para crescer por Figura 4 mostra como o muro está hoje em dentro do muro, contribuindo para a dia.

integração da estrutura no local e

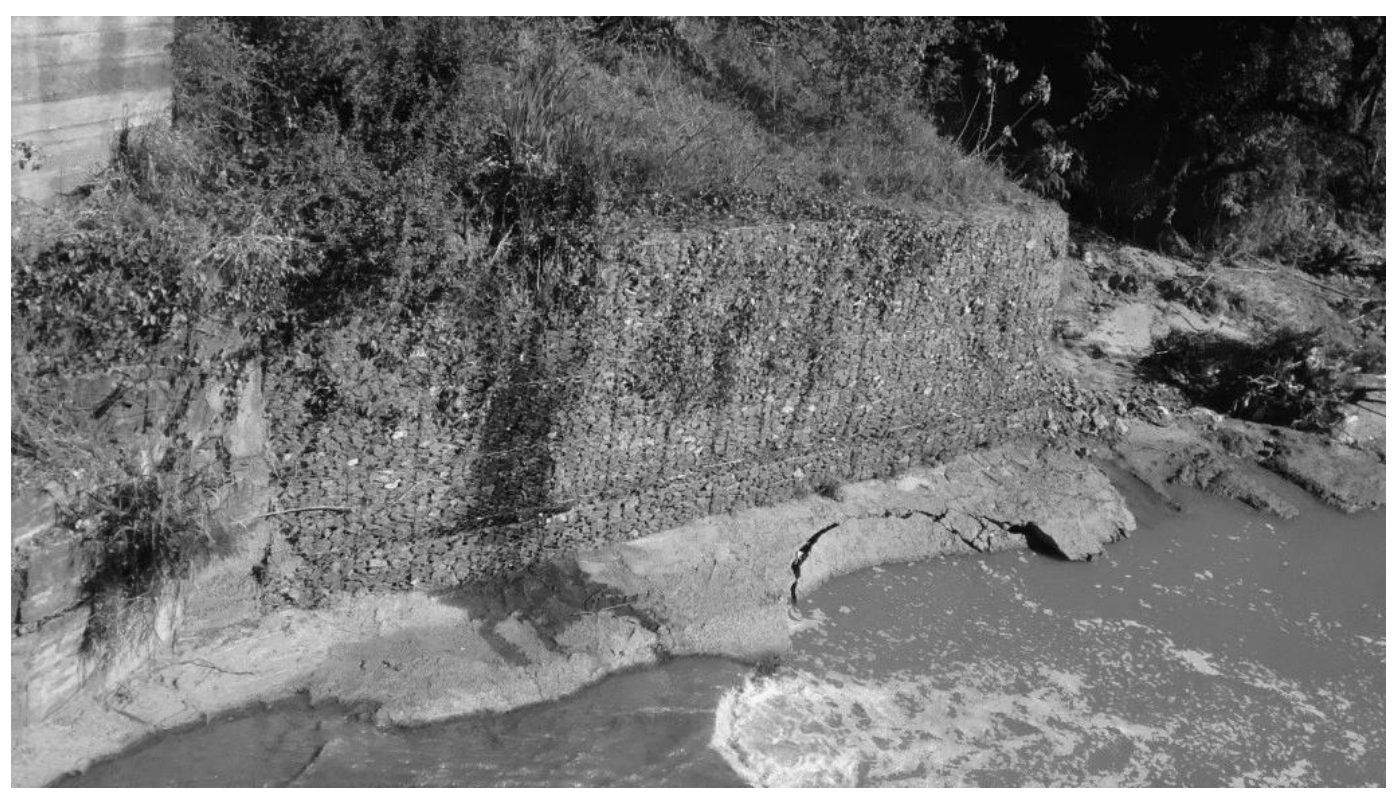

Figura 04. Muro de gabião no campus II da Universidade do Oeste Paulista.

Fonte: (Os autores, 2016) 
Para a elaboração de um muro no programa GEO5, nas verificações de estabilidade ao deslizamento e ao tombamento, é considerado um fator de segurança no valor de 1,5 .

A estrutura considerada para esse estudo, passou nos testes dos fatores de segurança ao tombamento com fator de segurança ao tombamento com $\mathrm{FT}=3,09$ e no fator de segurança ao deslizamento com FS= 1,88 , significando que ela é segura contra os empuxos ativos. Na Figura 5 mostra os valores da verificação realizada.

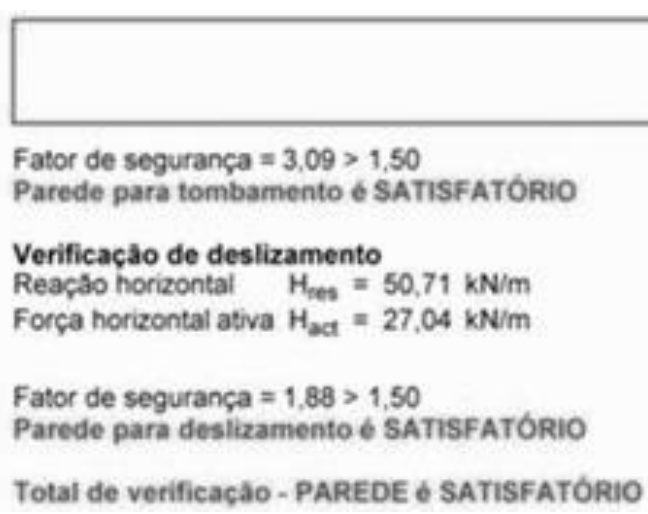

Figura 05. Resultado do dimensionamento do muro gabião.

Fonte: (GEO5, adaptado pelos autores, 2016)

$\mathrm{Na}$ análise do levantamento segundo lugar, com $\mathrm{R} \$ 397,72 \mathrm{o} \mathrm{m}^{3}$, e por quantitativo, o resultado mostrou que o último, o de alvenaria de pedra, por muro ciclópico é o mais barato, custando $R \$ \quad R \$ 486,110 \mathrm{~m}^{3}$.

347,66 o $\mathrm{m}^{3}$. Já o muro gabião aparece em 


\begin{tabular}{|c|c|c|c|c|c|c|}
\hline \multicolumn{7}{|c|}{ PLANULIMA ORÇAMENTÁRIA - MURO GABIĀO } \\
\hline ITEM & $\begin{array}{c}\text { COOIGO } \\
\text { SINAPI. } \\
\text { Preso } \\
1205 / 2016\end{array}$ & DISCRIMINACAOO & UNIDADE & QUANTDDADE & $\begin{array}{c}\text { PREÇO } \\
\text { UNITÁRIO }\end{array}$ & $\begin{array}{l}\text { PRECO } \\
\text { TOTAL }\end{array}$ \\
\hline \multicolumn{7}{|l|}{01} \\
\hline 01.01 & 92743 & $\begin{array}{l}\text { MURO DE GAEIAO, ENCHIMENTO COM PEDRA DE MATO } \\
\text { TIPO RACHALO, DE GRAVDADE. COM GAOLAS DE } \\
\text { COMPRIMENTO IGUALA } 2 \text { METROS, ALTURA DO MURO DE } \\
\text { ATE } 4 \text { METROS - FORNECIMENTO E EXECUCAO. }\end{array}$ & $m^{3}$ & 1,00 & 397.72 & 397,72 \\
\hline
\end{tabular}

\begin{tabular}{|c|c|c|c|c|c|c|}
\hline \multicolumn{7}{|c|}{ PLANILHA ORÇAMENTARIA - MURO CONCRE TO CICLOPICO } \\
\hline ITEM & $\begin{array}{c}\text { CODIGO } \\
\text { SINAPI - } \\
\text { Preso } \\
12 \text { Jos/2016 }\end{array}$ & DISCREMINACAO & UNIDADE & QUANTIDADE & $\begin{array}{l}\text { PRECO } \\
\text { UNITARIO }\end{array}$ & $\begin{array}{l}\text { PREÇO } \\
\text { TOTAL }\end{array}$ \\
\hline \multicolumn{7}{|l|}{01} \\
\hline 0101 & 738430001 & $\begin{array}{l}\text { MURO DE ARRIMO DE CONCRETO CICLOPICO COM } 30 \% \\
\text { DE PEDRA DE MAO. }\end{array}$ & $\mathbf{m}^{*}$ & 1,00 & 332,61 & 332,61 \\
\hline 01.02 & $74157 / 002$ & $\begin{array}{l}\text { LANCAMENTO E ADENSAMENTO DE CONCRE TO } \\
\text { INCLUINDO VIERACAOO }\end{array}$ & $\mathbf{m}^{*}$ & 1,00 & 15,05 & 15,05 \\
\hline \multicolumn{6}{|c|}{ TOTAL DO ITEM RS } & 347,66 \\
\hline
\end{tabular}

\begin{tabular}{|c|c|c|c|c|c|c|}
\hline \multicolumn{7}{|c|}{ PLANILHA ORCCAMENTÁRIA - MURO DE ALVENARIA DE PEDRA } \\
\hline ITEM & $\begin{array}{l}\text { COOIGO SINAPI } \\
\text { Preço 12/05/2016 }\end{array}$ & DISCRIMINACÄO & UNIDADE & QUANTDOADE & $\begin{array}{l}\text { PRECOO } \\
\text { UNITÁRIO }\end{array}$ & $\begin{array}{l}\text { PRECO } \\
\text { TOTAL }\end{array}$ \\
\hline \multicolumn{7}{|l|}{01} \\
\hline 01.01 & $73844 / 001$ & $\begin{array}{l}\text { MURO DE AFRIMO DE ALVENARIA DE PEDRA } \\
\text { ARGASASSADA. }\end{array}$ & $\mathrm{m}^{3}$ & 1,00 & 486,11 & 486,11 \\
\hline \multicolumn{6}{|c|}{ TOTAL DO ITEM RS } & 486,11 \\
\hline
\end{tabular}

Figura 06. Planilhas orçamentárias dos muros de contenção. Fonte: (SINAP, 2016)

\section{DISCUSSÃO}

O gabião também oferece algumas opções de utilização diferentes da que foram encontradas em Presidente Prudente, como por exemplo na arquitetura, aonde poderia ser integrado em projetos arquitetônicos com função estética, além de estrutural, sendo uma sugestão para projetos futuros na cidade.

Com o auxílio de softwares de geotecnia, se tornou mais fácil e rápido fazer verificações de cálculos de muros de contenções, sendo a maior responsabilidade do projetista a inserção dos dados corretamente e a interpretação concisa dos resultados obtidos.

Um dos quesitos fundamentais de qualquer tipo de obra de engenharia, é a questão econômica, se tornando o principal critério para a escolha de um método de construção. No entanto, deve-se avaliar o custo-benefício ao longo dos anos, levando em consideração as manutenções regulares, para assim definir qual será utilizado.

Em primeira avaliação, o muro de gabião parece ser menos econômico que o de concreto ciclópico, mas de acordo com Barros (2008), devido à flexibilidade da estrutura e rigidez de seus componentes, os muros gabiões podem suportar os desgastes 
naturais ao longo dos anos, desde que sua estrutura seja executada corretamente, e ainda permite com que a estrutura sofra movimentações acompanhando o recalque ou acomodações do terreno sem prejuízo da sua eficiência, ao contrário do muro de concreto ciclópico que são rígidos, não oferecendo a possibilidade de deformação, podendo sofrer mais com a ação de recalques e as movimentações.

\section{CONCLUSÃO}

O muro de contenção tipo gabião mostrou-se bastante ajustável ao meio ambiente. Como geralmente é preenchido com materiais naturais, sua adaptação é realizada facilmente a vegetação.

Além de ser usado como estrutura de contenção, também poderia ser aproveitado como parte estética em projetos de arquitetura em Presidente Prudente.

Os resultados do dimensionamento pelo programa GEO5 mostraram-se satisfatórios. Além de ser possível a elaboração de um modelo por um software, é possível ver se a estrutura suportará toda a carga atuante sobre ela, auxiliando o projetista durante a elaboração do projeto.

$\mathrm{Na}$ avaliação de custo, $\mathrm{O} \mathrm{m}^{3}$ do gabião foi o segundo mais barato, ficando atrás do concreto ciclópico. No entanto, com a análise da estrutura a longo prazo favorecem a escolha do gabião devido a suas características físicas.

\section{REFERÊNCIAS}

BARROS, P. L. A. Manual técnico de obras de contenção. 1. ed. São Paulo: Maccaferri do Brasil, 2008. 223p.

GEO5: análises geotécnicas. Version demo. [S.I]: Fine Civil Engineering Software, 2006.

GERSCOVICH, D. M. S. Estruturas de contenção muro de arrimo. 2007. Disponível em:

<http://www.eng.uerj.br/ denise/pdf/muros .pdf>. Acesso em: 15 maio 2015.

MACCAFERRI AMÉRICA LATINA. 2016.

MOLITERNO, A. Caderno de muros de arrimo. 1. ed. São Paulo: Blucher, 1980. $194 p$.

SINAP. Índices da construção civil. 2016.

Disponível em:

$<$ https://www.sipci.caixa.gov.br/SIPCI/servlet /TopController>. Acesso em: 12 maio 2016. 